Ultra-Fast Flash Observatory for the observation of early photons from gamma-ray bursts

This content has been downloaded from IOPscience. Please scroll down to see the full text. 2013 New J. Phys. 15023031

(http://iopscience.iop.org/1367-2630/15/2/023031)

View the table of contents for this issue, or go to the journal homepage for more

Download details:

IP Address: 161.111.165.153

This content was downloaded on 16/06/2016 at $11: 39$

Please note that terms and conditions apply. 


\title{
Ultra-Fast Flash Observatory for the observation of early photons from gamma-ray bursts
}

\author{
I H Park ${ }^{1,13}$, S Brandt ${ }^{2}$, C Budtz-Jørgensen ${ }^{2}$, A J Castro-Tirado ${ }^{3}$,

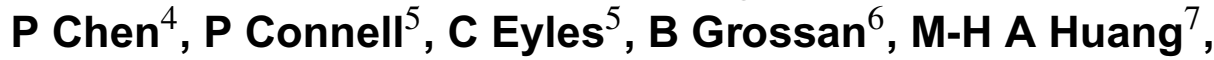

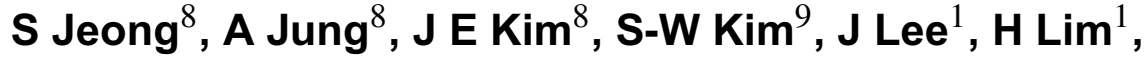 \\ E V Linder ${ }^{8,10}$, T-C Liu ${ }^{4}, \mathbf{K}$ W Min ${ }^{11}, \mathbf{G} \mathbf{W ~ N a}{ }^{8}, \mathbf{J} \mathbf{W ~ N a m}{ }^{4}$, \\ M I Panasyuk ${ }^{12}$, V Reglero ${ }^{5}$, J Ripa ${ }^{1}$, J M Rodrigo ${ }^{5}$, \\ G F Smoot ${ }^{8,10}$, S Svertilov ${ }^{12}$, N Vedenkin ${ }^{12}$ and I Yashin ${ }^{12}$ \\ ${ }^{1}$ Department of Physics, Sungkyunkwan University, 2066 Seobu-ro, \\ Suwon 440-746, Korea \\ ${ }^{2}$ National Space Institute, Astrophysics, Technical University of Denmark, \\ DK-2800 Kongens, Lyngby, Denmark \\ ${ }^{3}$ Instituto de Astrofisica de Andalucia (IAA-CSIC), PO Box 03004, E-18080 \\ Granada, Spain \\ ${ }^{4}$ Department of Physics, National Taiwan University, 1 Roosevelt Road, \\ Taipei 106, Taiwan \\ ${ }^{5}$ Universidad de Valencia, GACE, Edif. de Centros de Investigacion, Burjassot, \\ E-46100 Valencia, Spain \\ ${ }^{6}$ Space Sciences Laboratory, University of California at Berkeley, USA \\ ${ }^{7}$ Department of Energy Engineering, National United University, 1, Lienda, \\ 36003 Miaoli, Taiwan \\ ${ }^{8}$ Department of Physics, Ewha Womans University, 11-1 Daehyun-dong, \\ Seoul 120-750, Korea \\ ${ }^{9}$ Department of Astronomy, Yonsei University, 134 Shinchon-dong, Seoul \\ 120-749, Korea \\ ${ }^{10}$ Institute for the Early Universe, Ewha Womans University, 11-1 \\ Daehyun-dong, Seoul 120-750, Korea
}




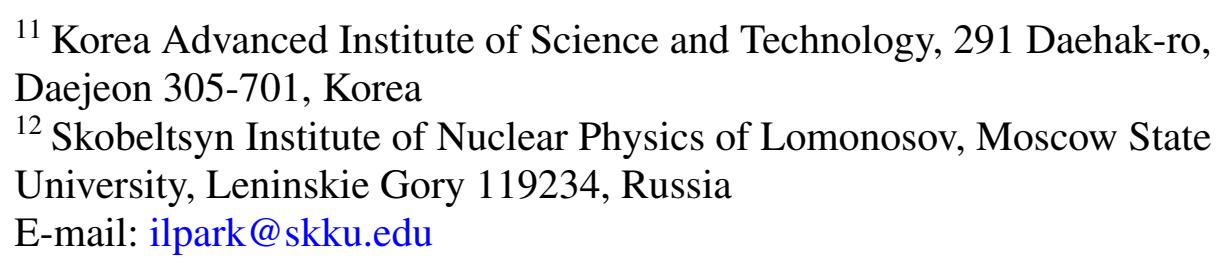

New Journal of Physics 15 (2013) 023031 (21pp)

Received 2 October 2012

Published 19 February 2013

Online at http://www.njp.org/

doi:10.1088/1367-2630/15/2/023031

\begin{abstract}
One of the least documented and understood aspects of gamma-ray bursts (GRBs) is the rise phase of the optical light curve. The Ultra-Fast Flash Observatory (UFFO) is an effort to address this question through extraordinary opportunities presented by a series of space missions including a small spacecraft observatory. The UFFO is equipped with a fast-response Slewing Mirror Telescope (SMT) that uses a rapidly moving mirror or mirror array to redirect the optical beam rather than slewing the entire spacecraft to aim the optical instrument at the GRB position. The UFFO will probe the early optical rise of GRBs with sub-second response, for the first time, opening a completely new frontier in GRBs and transient studies. Its fast response measurements of the optical emission of dozens of GRBs each year will provide unique probes of the burst mechanism and test the prospect of GRBs as a new standard candle, potentially opening up the $z>10$ universe. For the first time we employ a motorized slewing stage in SMT that can point to the event within $1 \mathrm{~s}$ after the $\mathrm{X}$-ray trigger provided by the UFFO Burst Alert and Trigger Telescope. These two scientific instruments comprise the UFFO-pathfinder payload, which will be placed onboard the Lomonosov satellite and launched in 2013. The UFFOpathfinder is the first step of our long-term program of space instruments for rapid-response GRB observations. We describe early photon science, our soonto-be-launched UFFO-pathfinder hardware and mission, and our next planned mission, the UFFO-100.
\end{abstract}

${ }^{13}$ Author to whom any correspondence should be addressed. 


\section{Contents}

1. Introduction 3

2. Current limits of rapid response measurements 5

3. Slewing Mirror Telescope $\quad 8$

4. The science case for prompt response UV/optical observations 9

4.1. Early rise of light curves . . . . . . . . . . . . . . . . . . 10

4.2. Short-duration gamma-ray bursts . . . . . . . . . . . . . . . . 10

4.3. Dark gamma-ray bursts . . . . . . . . . . . . . . . . . . . . 11

4.4. Physical time scales in compact objects . . . . . . . . . . . . . . 11

4.5. Association of emission processes by cross-correlations . . . . . . . . . . . . 11

4.6. Association of emission processes by spectral slope . . . . . . . . . . . . . 11

4.7. Test of shock models with the bulk Lorentz factor . . . . . . . . . . . . . . 12

4.8. Identification of internal shock via fast variability . . . . . . . . . . . . . . 12

4.9. Tie-in to compact objects and gravitational wave observations . . . . . . . . . 12

4.10. Time evolution of local dust . . . . . . . . . . . . . . . . 13

5. The Ultra-Fast Flash Observatory (UFFO) program 13

6. UFFO-pathfinder 14

6.1. Slewing Mirror Telescope of UFFO-pathfinder . . . . . . . . . . . . . . . . . . 14

6.2. The UFFO Burst Alert and Trigger Telescope of UFFO-pathfinder . . . . . . . 15

6.3. The UFFO-pathfinder data acquisition . . . . . . . . . . . . . . 16

7. UFFO-100 17

8. Summary 19

Acknowledgments 19

$\begin{array}{lr}\text { References } & 20\end{array}$

\section{Introduction}

Gamma-ray bursts (GRBs) are the most luminous explosions in the universe, emitting the highest energy photons, and are detected to the highest red-shift of any discrete source in the universe [1]. These properties provide great leverage in time, wavelength and information and thus a unique opportunity to understand not only the nature of the universe but also fundamental physics, to name but a few, as listed in the following.

GRBs allow the study of the evolution of stars and stellar populations from red-shifts $z=0-15$ [2] or over $98 \%$ of the age of the universe. The first stars and galaxies at $z=10-15$ would be identified with their formation history [3], and the reionization history at $z \sim 7$ could be unveiled as GRBs would be signposts of the faint galaxies providing the bulk of UV photons to reionization of the universe [4]. GRBs could be a potential candidate for the next generation cosmological standard candles [5-7], although not reaching a general consensus [8]. If such a luminosity calibration is realized, GRBs could probe the early universe at red-shift values of up to $z=15$. It would be nothing less than a revolution in extremely high- $z$ cosmology, as other cosmological tools (supernovae (SNe) Ia [9], baryon acoustic oscillations [10] and lensing [11]) are much more limited in $z$ range for the foreseeable future. 
GRBs offer a great opportunity to explore the extreme universe. Among the many other avenues of research are: the origin of GRBs including short GRBs [12] and dark GRBs [13]; fast transient cosmic flash events such as primordial black holes [14]; black hole event horizon; tidal disruption [15] and shock break-out [16] associated with extremely heavy gravitational objects. GRBs are believed to be sources of ultra-high energy cosmic rays (UHECR) [17] and gravitational waves (GW) [18]. GRB emission spans some nine orders of magnitude in photon energy, a prime opportunity for synoptic observations. In addition, GRBs are a prime example of 'multi-messenger' astrophysics as photon measurements are augmented with observations of UHECR particles through air showers (e.g. TA [19], Auger [20], TUS [21] and JEMEUSO [22]) and neutrinos (e.g. ICECUBE [23] and ANITA [24]), and searches for GW (e.g. LIGO [25] and LISA/NGO [26]). For GRBs, rapid response is required to recover the rich information in both the individual processes giving rise to each 'messenger' and the time delay between them.

GRBs have been observed from space and studied extensively over the last few decades, e.g. by the successful CGRO instrument [27], which captured almost 3000 observations of GRBs and with its four instruments reached a sensitivity over the wide energy range from $30 \mathrm{keV}$ to $30 \mathrm{GeV}$. Another breakthrough mission was the BeppoSAX satellite [28] providing accurate localization of GRBs at the order of a few arcmin in the x-ray range along with observations in the gamma range and fast distribution of the GRB coordinates to ground-based observations within a few hours. After HETE-2 [29], several missions including Integral [30], Swift [31] and Fermi [32] were launched in 2002, 2004 and 2008, respectively, and are currently in operation in space.

In spite of the wide knowledge already acquired about GRBs there are still many open questions about their progenitors and environment. A deeper understanding of GRBs requires more statistics of GRBs, particularly of high- $z$ GRBs, measurements of polarization and a wider range of not only spectral coverage extending to sub-keV x-ray and infrared (IR) but also temporal coverage through rapid pointing of telescopes at GRBs. These observational capabilities are to be incorporated in future missions. They are expected to enlarge the detection area and/or volume of x-rays and the aperture of UV/optical/IR telescopes, and to improve photometric, temporal and spectral sensitivities as well. Moreover, Swift is very unlikely to be able to extend its operations much longer than its designed lifetime. Post-Swift missions are foreseen, as well as missions primarily dedicated to the subjects above, i.e. SVOM [33] and JANUS [34] for high- $z$ measurements, POLAR [35] for prompt gamma polarimetry and UFFO series for early photon measurements.

Thorough understanding of GRBs and their underlying physics will be aided by multiwavelength observations in the early emission phase. Hundreds of GRB UV/optical light curves have been measured since the discovery of optical afterglows [36]. The Swift spacecraft, the fastest high-sensitivity space observatory for following the UV/optical afterglows, has simultaneously measured x-ray and UV/optical signals in hundreds of GRBs [31]. However, after nearly 8 years of operation of Swift, the immediate aftermath of the explosion is scarcely observed in the optical or UV, because the Swift instrument typically responds in $\sim 100$ s or more. Ground-based telescopes do occasionally respond faster, but only a relatively small number of rapid detections have been produced to date with heterogeneous sensitivities and other characteristics. Only a few short-duration GRBs have been detected in the UV/optical/IR within the first minute after the gamma-ray signal. 
This lack of early observations and the blindness to the rise phase of many GRB optical light curves along with those of other rapidly variable transient sources leaves fertile astrophysical territory. Many important physical questions arising at the short time scales remain unexplored. Rapid data collection is also essential for tests of fundamental physics such as constraints on Lorentz violations [37] and CPT [38] from the time delay between different energy photons, or between photons and neutrinos. Coincident or successive observations of the explosion event as an electromagnetic counterpart to a neutrino or gravitational wave observatory signal would revolutionize astronomy, and greatly improve our understanding of black holes, neutron stars and strong field gravity.

We have developed methods, for the first time, for reaching sub-minute and sub-second time scales in a spacecraft observatory appropriate for launch even on small satellites. Rather than slewing the entire spacecraft to aim the optical instrument at the GRB position, we have proposed a Slewing Mirror Telescope (SMT) that employs a rapidly moving mirror or mirror arrays to redirect the optical path at a telescope. We describe in the following the concept and development of a fast-response optical telescope, the early photon physics with the UFFO project $^{14}$, the current status of the first mission UFFO-pathfinder [39] onboard the Lomonosov spacecraft to be launched in 2013, and a proposed full-scale mission of UFFO-100 as the next step.

\section{Current limits of rapid response measurements}

The Swift observatory obtains UV/optical light curves by first serendipitously detecting the onset of a GRB within the very large field of the Burst Alert Telescope (BAT) [40]. The BAT then produces a crude sky position via a standard coded mask technique. After this, the entire observatory spacecraft slews to point the UV/optical telescope (UVOT) and other instruments at the GRB position. After slewing, a period of time is required for the pointing to stabilize, after which a series of UVOT exposures begins. Although this system has been a great success by producing numerous detections of optical afterglows associated with GRB, only a handful of responses have occurred in less than $60 \mathrm{~s}$. Figure 1 shows the number of UV/optical events versus the response time of UVOT after the trigger with BAT since GRB050318. The response frequency falls off for response time below $100 \mathrm{~s}$ with an almost complete cutoff by $60 \mathrm{~s}$. Due to its finite mission lifetime, Swift cannot be expected to significantly increase this number of sub-minute responses.

The position calculated by Swift within $5-7 \mathrm{~s}$ is also broadcast over the internet via the gamma-ray coordinate network $\left(\mathrm{GCN}^{15}\right)$. At this point, any instruments may respond and follow up the coordinates in the easily-machine-readable format of the GCN alerts, via email or socket connection. Although the response of some instruments on the ground (to name a few: ROTSEI-III [41], RAPTOR [42], PAIRITEL [43], Super-LOTIS [44] and BOOTES [45]) is extremely rapid, e.g. $25 \mathrm{~s}$ for ROTSE-III, the sensitivity is far less than that of the Swift UVOT. Due to their small size and to the limitations of ground-based observing including daytime and weather, together these instruments have managed only a handful of rapid detections [46]. A concurrent optical and gamma observation of the prompt phase of GRB080319B [47] was achieved luckily by TORTORA [48] on the REM telescope ${ }^{16}$ and by 'Pi of the Sky' [49] when this GRB occurred

\footnotetext{
${ }^{14}$ http://uffo.skku.edu

$15 \mathrm{http}: / / g c n . g s f c . n a s a . g o v$

16 http://www.rem.inaf.it
} 


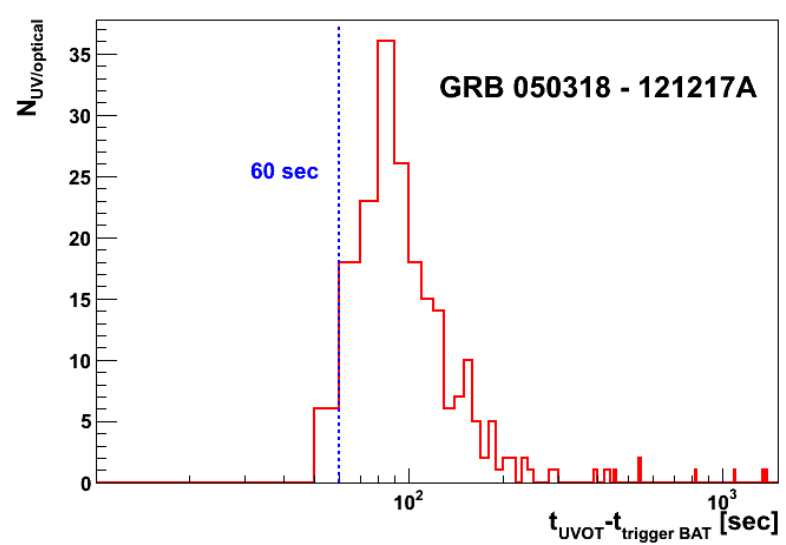

Figure 1. The detected number of Swift's UV/optical events versus the response time of UVOT since 2005.

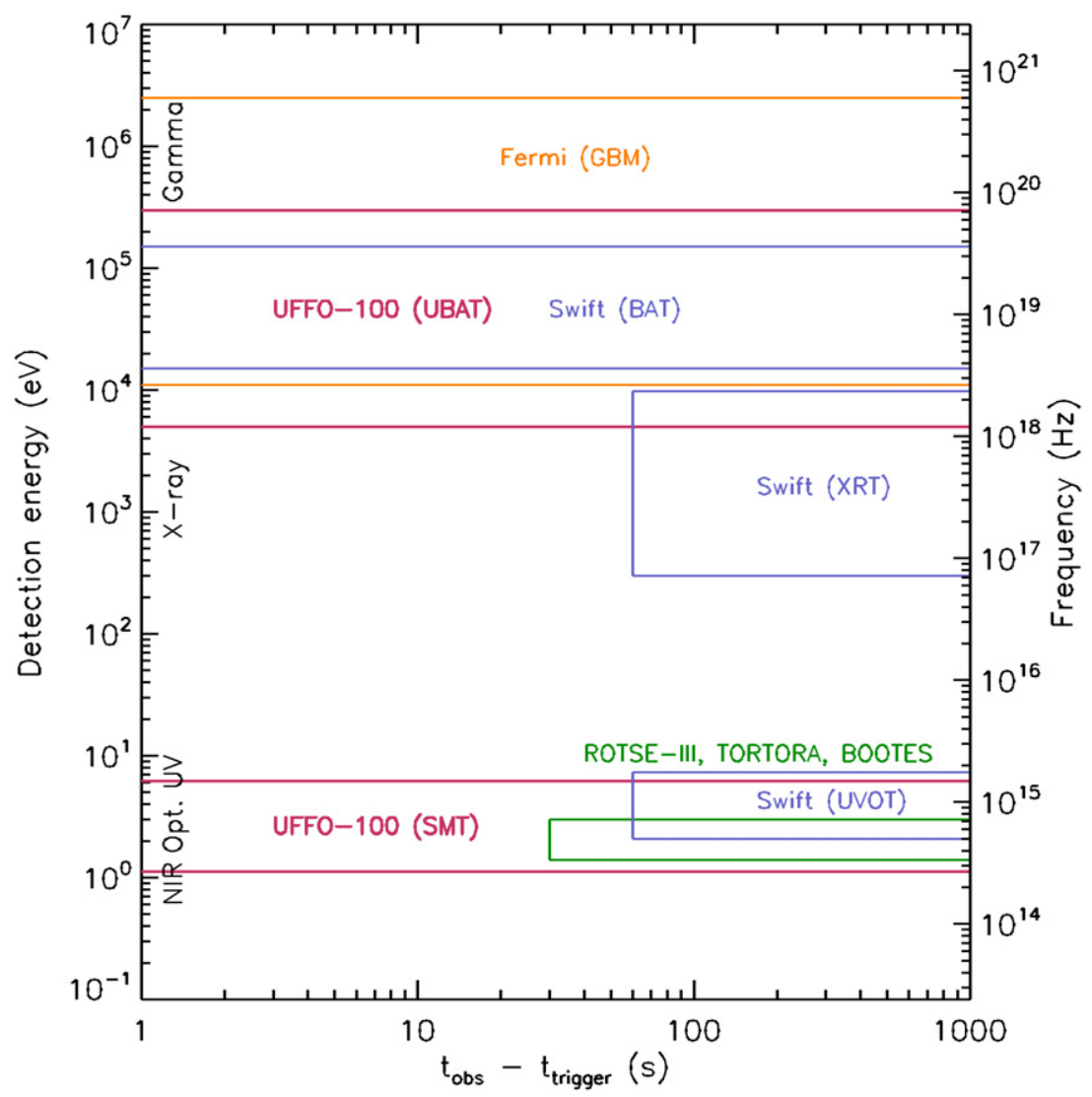

Figure 2. The domain of frequency and time accessible by space and ground experiments. The UFFO missions will explore the fast- and ultra-fast regimes below $60 \mathrm{~s}$ and even below $1 \mathrm{~s}$, in a systematic survey.

in their field of view (FOV) and in the FOV of the Konus/Wind instrument [50]. Figure 2 shows the domain of frequency and time accessible by space and ground experiments. The UFFO missions will explore the blank parameter space, the fast- and ultra-fast regimes below $60 \mathrm{~s}$ and even below $1 \mathrm{~s}$, in a systematic survey and thus significantly enlarge the sample of 


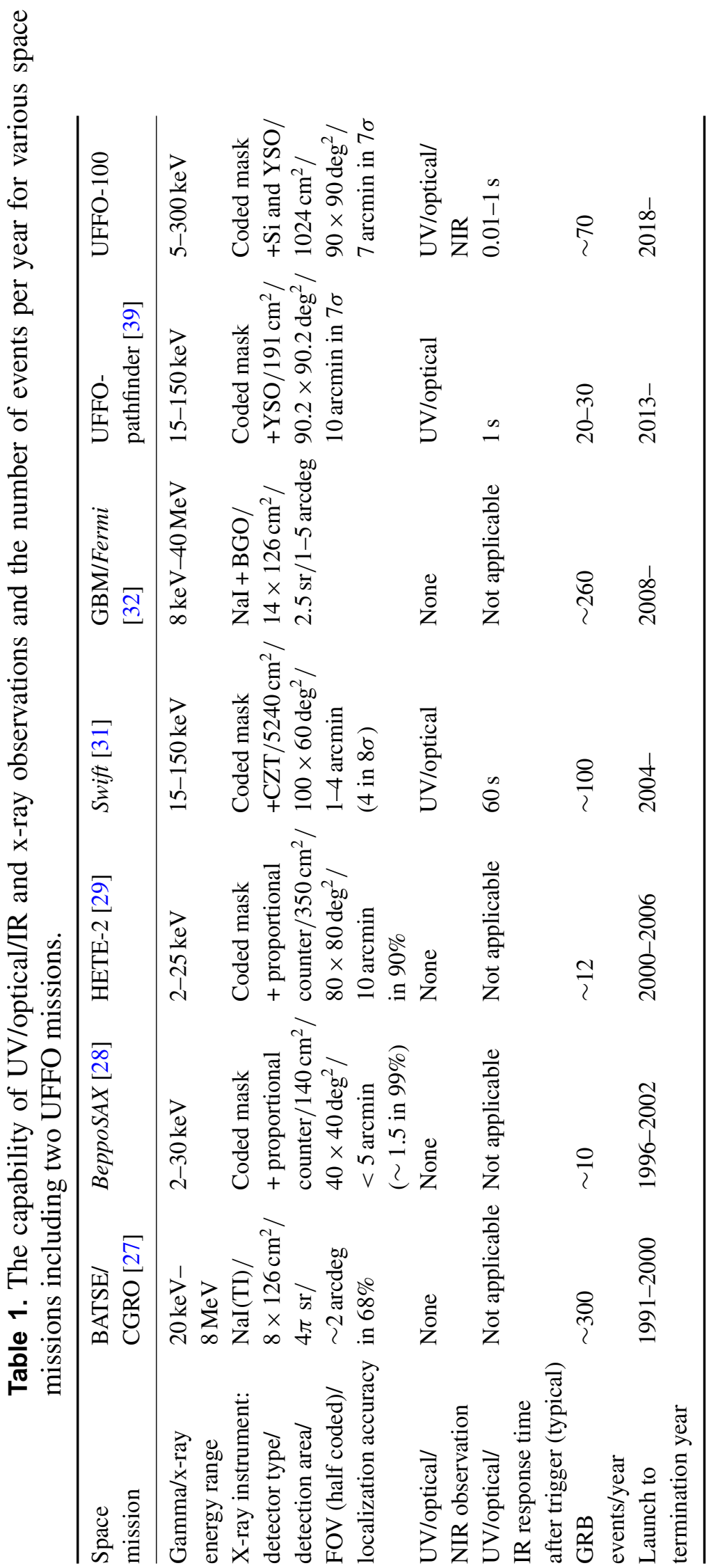




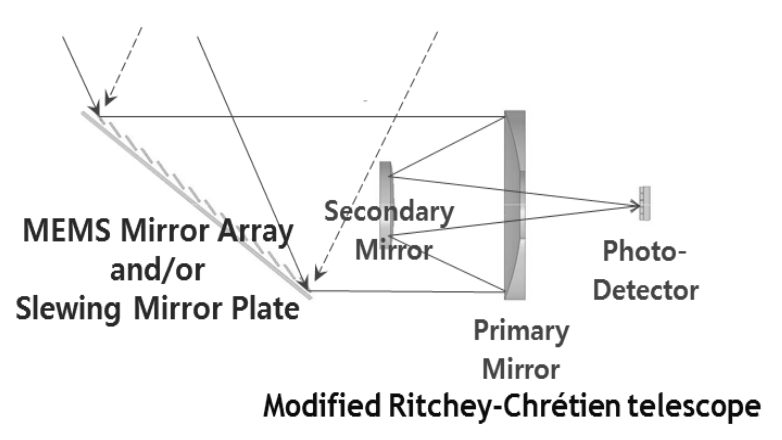

Figure 3. Schematic diagram of the SMT's beam redirection system.

such observations. Table 1 lists the capability of UV/optical/IR and x-ray observations and the number of events per year for various space missions including two UFFO missions.

Because of the ability of space-based telescopes to detect photons throughout the UV/optical band without atmospheric scattering or absorption, the $30 \mathrm{~cm}$ aperture UVOT telescope compares favorably in sensitivity to a $4 \mathrm{~m}$ ground-based telescope [51], and such telescopes are not capable of sub-minute response. The slower slew times of such larger terrestrial telescopes make them uncompetitive for the sub-1000 s regime. The Swift limit of $60 \mathrm{~s}$ response is therefore the practical minimum for sensitive UV/optical GRB studies for the near to mid-term future. In the following, we propose a new technical solution to overcome the constraints of current instrumentation.

\section{Slewing Mirror Telescope}

After triggering, conventional GRB observatories in space or on the ground must reorient their entire spacecraft or telescope to aim their narrow field instruments at the GRB. The time to rotate the spacecraft to slew the UVOT is the limiting factor in the Swift response time; not only must the entire spacecraft mass be rotationally accelerated and decelerated, but also, after the movement, some additional time is required for any vibrations to cease.

Our approach to accelerating the slew capabilities is to redirect the optical path at an astronomical telescope via a substantially more lightweight slewing mirror rather than move the entire payload or telescope [39]. The slewing system can be either a flat mirror or mirror arrays such as the micro-electro-mechanical systems (MEMS) mirror array (MMA), mounted on a gimbal platform. In either case, a large FOV is accessible without the aberration inherent in wide-field optical systems.

Figure 3 shows the concept of SMT. Parallel rays from a distant source are directed onaxis with respect to the fixed optics by the moving mirror system. The net effect is to steer the UV/optical instrument beam, instead of moving the telescope or the spacecraft itself. The beam can be steered by two-axis rotation of the mirror plate, rotation of the individual MMA devices, or rotation of MMA and also gimbal afterwards.

We find that various types of rotating mirrors move across the entire FOV wider than $180^{\circ} \times 180^{\circ}$, point and settle in less than $1 \mathrm{~s}$. In order to build a telescope with milliseconds slew speed both for the $x$ - and $y$-directions at a time, our laboratory consortium has produced small mirror arrays driven by MEMS devices. Resembling mirror segments mounted on twoaxis gimbals, MEMS micromirrors are fabricated in arrays using advanced silicon and integrated circuit technologies. These MEMS mirror arrays, fabricated like other microelectronics devices, can move, point and settle in less than a few ms with rotation angle $\pm 15^{\circ}$ off axis and thus 


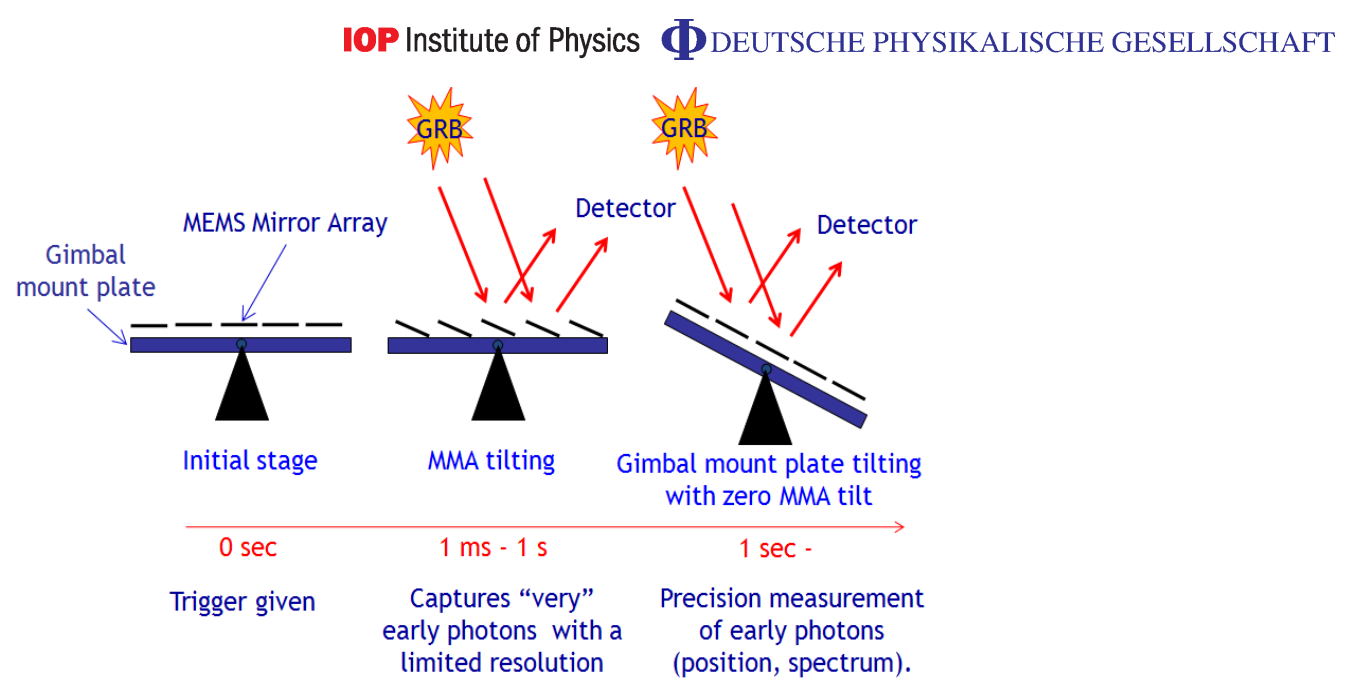

Figure 4. In the ultra-fast mode, the gimbal mount plate remains aimed at the middle of the field, while the MMA elements point at the target. In the fast mode, the MMA elements are in standby or zero deflection mode, and the plate aims at the source. The first mode minimizes response time, the second maximizes sensitivity. The MMA provides ultra-fast response times, but at some cost in terms of PSF broadening. There is essentially no degradation in performance for the MMA in standby mode, and the full resolution (and therefore sensitivity) of the telescope is obtained in fast mode.

FOV of $60^{\circ} \times 60^{\circ}$. Only voltages are applied to tiny electric actuators for rapidly pointing to observe bursts. These are extremely lightweight and low-power devices that are well suited to the platform of a microsatellite. A series of small prototype MMA systems has been developed in our group since 2004 [52]. We fabricated a small prototype of a $3 \mathrm{~mm}$ caliber telescope to demonstrate the idea of fast slewing or tracking [53]. It was flown once in space on the ISS in 2008, and once on Tatiana-2 satellite in 2009, with excellent performance, both for nadir observation of transient luminous events occurring in the upper atmosphere [54].

Simulations of our segmented MMA show that the point spread function (PSF) of SMT will have a full-width at half-maximum (FWHM) of about 1 arcsec with the micromirrors at zero tilt. When the micromirrors are tilted, however, the PSF spreads to an FWHM of 2 arcmin due to the difference in beam path length created by the tilt of elements. Therefore, we could use the MMA to steer the beam to measure very early photons, starting $\sim 10^{-3} \mathrm{~s}$ after trigger+location. In this ultra-fast mode, the mirror plate remains aimed at the middle of the field as shown in figure 4. We could perform high-resolution imaging of the source later, using the mirror plate to steer the beam (with the MMA at zero tilt). This fast mode motion takes much longer, $\sim 1 \mathrm{~s}$. So, the ultra-fast mode with MMA minimizes response time, while the fast mode with rotating plate maximizes sensitivity.

\section{The science case for prompt response UV/optical observations}

Beyond the possible physics with GRBs mentioned in section 1, the SMT offers a unique opportunity to probe a new, very early emission parameter space to thoroughly investigate the rise phase of GRBs, which so far has been observed only occasionally. A variety of rise time physics is given below. 


\subsection{Early rise of light curves}

The discovery of optical afterglows of GRB was a monumental event in modern astrophysics [55], ending the 30 year mystery of the GRB distance scale. The study of GRB UV/optical afterglows and their host galaxies has led to knowledge of the origin of some types of GRB and the discovery of the most distant explosions known (e.g. GRB 090423 at $z=8.2$ ) [56]. Much progress has been made in GRB science since the launch of the Swift observatory in 2004 [31]. The observations from Swift did not produce a simple picture of GRB, but rather documented the richness and complexity of this phenomenon. After some 370 UV/optical observations were made by Swift UVOT, a huge variation in light curves has been observed, especially in the early rise time. There appear to be distinct classes of fast-rising $\left(t_{\text {peak }}<10^{2} \mathrm{~s}\right)$ and slow-rising bursts [7]. Additionally, the light curves are complex, with decays, plateaus, changes in slope and other features that are not yet understood.

It is claimed [7] that among the population of GRBs with fast-rising optical light curves, the optical luminosity correlates with the rise time, giving promise as a kind of 'calibrated standard candle' much like SNe Ia, which would make GRBs useful as a cosmological probe of the very high red-shift universe. In order to move this possibility to the status of a refined tool, a larger sample of such optically fast-rising GRBs is required and, in particular, a better time resolution is required early on. Fastest-rising bursts often have none or just one measurement in their rising phase - hardly enough to understand the physics in this regime - and many other bursts have no early measurements at all. Fewer than ten GRBs in this study were measured at $<100 \mathrm{~s}$ after their burst trigger and not a single measurement was made at $<15 \mathrm{~s}$ after trigger.

In this respect, several fundamental questions arise. Are there more features in the early light curve that have been missed by such sparse sampling? Does any feature of the rise correlate with the luminosity or a particular aspect of the physics? How many bursts are misclassified because the rapid rise was missed? The need for earlier measurements (faster UV/optical response after the initial GRB) is clear and compelling.

\subsection{Short-duration gamma-ray bursts}

It has become apparent that there are several types of GRBs. First, GRBs have an obvious (but overlapping) separation on the spectral hardness versus duration plane, so the basic taxonomy separates these into short and hard type (SHGRBs) less than $\sim 2 \mathrm{~s}$ in duration and long and soft type (LSGRBs), more than $\sim 2 \mathrm{~s}$ in duration [57]. The short time scale of SHGRB emission, the associated lower luminosity and shorter time scale of the x-ray and optical afterglow lead to speculation that the two classes have fundamentally different physical origins [58]. LSGRBs are thought to originate from the collapse of massive stars, e.g. the collapsar model [59], and SHGRBs from the merger of compact objects such as neutron stars and black holes (for a review of SHGRBs, see, e.g., [60]). Other types of classifications, including those with more of a physical than phenomenological motivation, have been proposed (e.g. [61]). Very short GRB (VSGRB) may originate from the evaporating primordial black holes [14].

The recent progress in SHGRBs is extremely exciting. At the time of writing this, however, only about $\sim 20$ SHGRBs have had UV/optical measurements, often with only one measurement above background, and thus suffer from poor time resolution in their light curves. Two measurements during the decay period are required to determine the most rudimentary decay time constant, assuming a power-law decay. The rise phase of SHGRB optical emission is not observed in most cases. What is the shape of the rise? Is the shape homogeneous? 
The rise time may give rich information including the size of the system and the surrounding environment. The physical origin of this type of burst remains an outstanding mystery, so any hints on this origin would be extremely valuable. Is there any prompt UV/optical emission from such events? What would we see if we observed more of these events in the sub-minute or sub-second regime? Are there ultra-short events on the accretion disc dynamical time scale of compact objects (that are beamed so we can see them)? Earlier observations would answer these questions and open a new window probing compact object structure, populations and evolution.

\subsection{Dark gamma-ray bursts}

'Dark' GRBs are those that stand out as having a very faint optical signal compared to x-ray afterglow. It is only recently that extinction has been found to be the dominant source of dark GRBs [62]. An alternative scenario, however, suggests that some dark GRBs are simply due to a faster decay for optical than x-ray emission [63]. In this scenario, the optical emission fades in less than $\sim 10^{2} \mathrm{~s}$, so that most observations would not detect the optical afterglow. Better short time scale observations would shed light on this two-mechanism model.

\subsection{Physical time scales in compact objects}

In a more general sense, resolving the light curve peak time at any epoch gives a hint of the most important physical processes in that epoch. Coalescence of neutron star and black hole systems are features of a number of GRB models, particularly models for the less understood short GRB. The light crossing time of outer accretion disc bounds, the dynamic time scales of large accretion disc systems and other time scales are in the sub-minute regime, requiring a rapid response for their measurement. The time scales of jet formation or deceleration in these smaller systems may also be in this time regime.

\subsection{Association of emission processes by cross-correlations}

Another general tool that rapid-response observations afford is the correlation of light curves from different bands. If complex light curves in different bands have a clear correlation, this is a very strong argument for a physical linkage between the processes of emission in the two wave bands. The delay between the light curves gives further information about both processes [47]. Referring to the correlation of rapid-response light curves accidentally observed earlier, it is intriguing that early UV/optical light curves show good correlation with their gamma-X light curves (GRB041219 [64] and GRB080319B [47]), yet others do not (GRB990123 [64]). Is this a clue to additional processes, or a hint that the origin of these GRBs is quite different, i.e. SN Ia versus Ib? What will we see if we can extend these correlations of early emission to SHGRB?

\subsection{Association of emission processes by spectral slope}

The broad-band spectra of GRBs can be modeled by power laws evolving in time. Chromatic and achromatic jet breaks are important predictions/distinguishing features of models. One feature is the well-known transition from relativistic to non-relativistic emission, the transition from 'prompt' emission to afterglow. The change in spectral slope, and the time of this change, are therefore important diagnostics of the interaction of the jet and the surrounding medium, and/or injection of additional energy into the jet. The broad-band spectral slope itself is a 


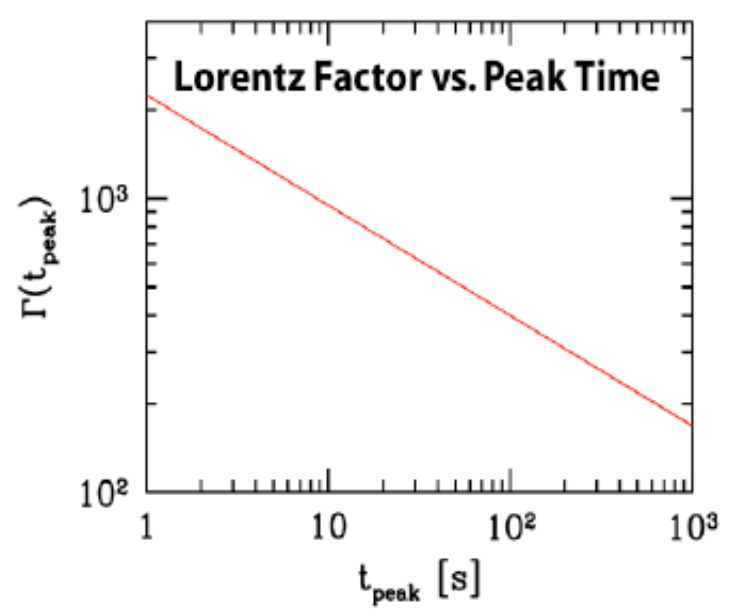

Figure 5. The bulk Lorentz factor versus peak emission time, using the method proposed in [65]. The observation of peak emission time provides the limits of the bulk Lorentz factor that constrains central engine models.

discriminator of the electron energy distribution, magnetic field and other features of the emission mechanism.

\subsection{Test of shock models with the bulk Lorentz factor}

Measurement of early UV/optical emission can serve as a probe of the physical conditions in the GRB fireball at short times. A simple, nearly model-independent argument [65] shows that the bulk Lorentz factor depends on the time of the early UV/optical emission peak. Measurement of the peak will therefore provide a measurement of the bulk Lorentz factor, as shown in figure 5.

\subsection{Identification of internal shock via fast variability}

At present, UV/optical emission at early times in typical bursts is believed to come from external shocks, and is predicted to have a smooth, monotonic rise (see, e.g., [66] and references therein). Observation of an early time UV/optical light curve that more closely resembles a gamma-X light curve, jagged and with multiple peaks, would clearly indicate the presence of a prompt optical emission produced by internal shock. Sub-minute measurements would be required to learn more about such prompt emissions.

\subsection{Tie-in to compact objects and gravitational wave observations}

The coming generation of gravitational wave observatories should regularly detect the coalescence of binary compact systems, the favored scenario for SHGRBs. This will open an entirely new field of astronomy. Because of its novel nature, corresponding UV/optical measurements will be highly important to interpret the astrophysics of the event. Moreover, although gravitational wave signals have potential to yield highly accurate distance measurements, they alone cannot break the degeneracy in parameters to yield the red-shift-that requires observation of an electromagnetic counterpart such as the GRB. Fast-response optical observations can test Lorentz violations from the time delay between different energy photons, 
or between photons and neutrinos or early emission with GW. Such a fast response would be essential for gaining a deep understanding of compact objects and cosmology [67].

\subsection{Time evolution of local dust}

Long-type GRBs are associated with massive stars and therefore offer a unique view of young star formation regions. Many authors have discussed GRB observations as an independent view of star formation rates and distribution in the universe [68]. Another characteristic of young star formation regions is the presence of dust, and indeed, many GRBs show optical extinction [69]. GRBs have more than enough hard photons to dissociate dust over large fractions of starforming regions [70]; this process is thought to take place on time scales less than or of the order of minutes [71], and would result in rapidly changing extinction. Measuring variable extinction via multi-band IR/optical observations therefore promises a unique tool for the study of local, star-formation-related dust, independently of host galaxy disc dust [72].

\section{The Ultra-Fast Flash Observatory (UFFO) program}

The UFFO is to pioneer the hitherto unexplored time domain nature of GRB by using the concept of SMT's fast or ultra-fast slewing mirror technology. It will respond to initial photons within a fraction of a second after the burst of GRB. The UFFO project will be carried out in a series of relatively light payloads to be adaptable readily to micro or small satellites.

One peculiar property of GRBs renders relatively small x-ray trigger instruments possible; it is the GRB distribution in flux. Typical astrophysical sources have a number count versus flux distribution close to a uniform Euclidian distribution, with a sharp cutoff caused by the sensitivity of the instrument. GRBs, in contrast, are so bright that they are limited by the volume of the observable universe. Star formation, rather than the sensitivity of our largest instruments, is the limiting factor. This means that for the simple location of a GRB (not a detailed study of the x-ray through the gamma-ray spectrum), a small instrument can locate a large fraction of those detected by much larger instruments. This property of GRBs makes the UFFO concept possible [39].

The first payload of UFFO is UFFO-pathfinder. It will be flown aboard the Lomonosov spacecraft in 2013. Although the pathfinder is small and limited, it could serve as the observational cornerstone of future mission development for rapid responses. Indeed, the GRB detection rate of UFFO-pathfinder will be about $60 \%$ of Swift for the same type of orbit, and the sub-minute measurements of the UV/optical emission of dozens of these GRBs each year would provide the first detailed measurements of fast-rise GRB optical light curves. The next serious version, UFFO-100, is expected to be launched in 2018, improving on the UFFO-pathfinder with a relatively modest size. The UFFO-100 will extend its measurement capability to near-IR (NIR) using a dichroic beam splitter on the SMT optics bench.

In this decade, Swift will likely cease operations, and the UFFO will continue to provide GRBs for follow-up studies by the optical ground-based multi-wavelength communities, providing future possibilities for understanding GRBs of all types, and giving hope for their use as extreme- $z$ cosmological probes. If other Swift-like GRB missions were to fly in the near future, their sensitivity would complement the UFFO's rapid response. 

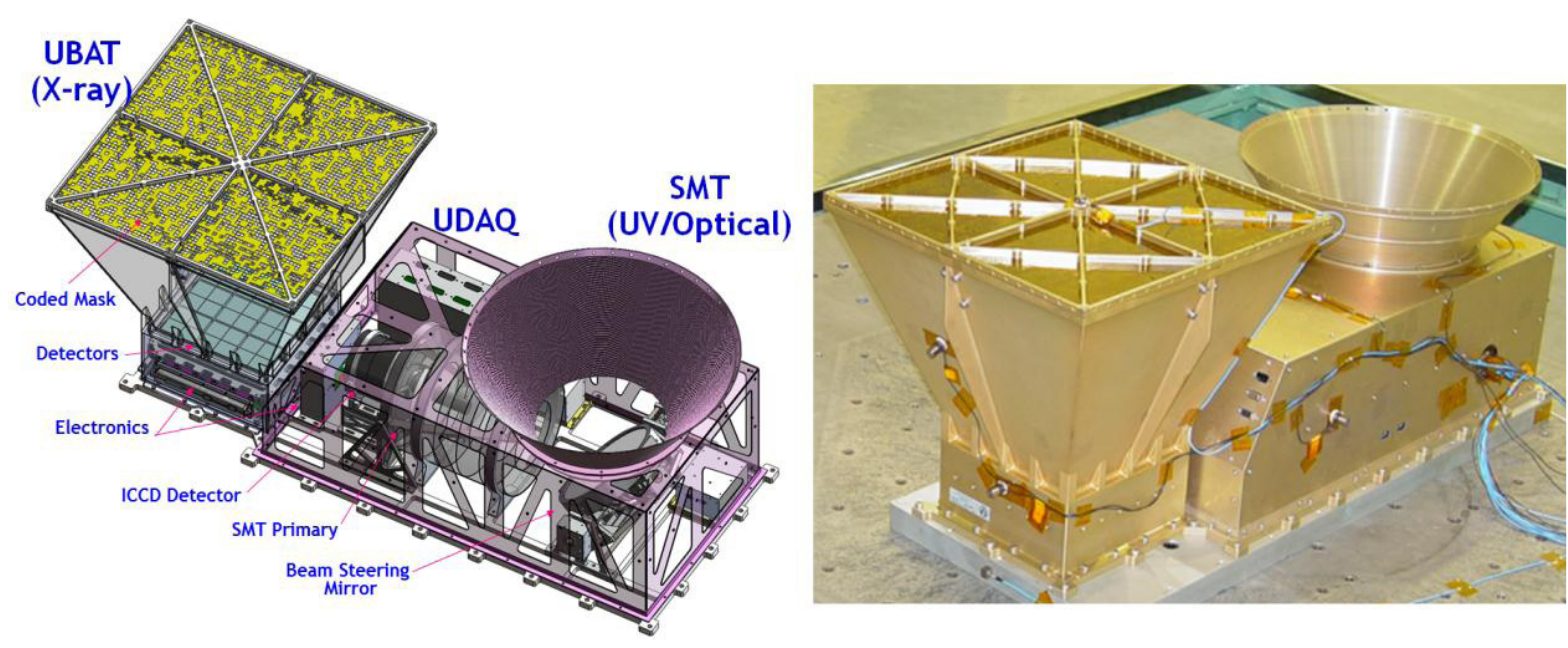

Figure 6. A rendering of the integrated UFFO-pathfinder (left) and the fabricated flight model (right).

\section{UFFO-pathfinder}

The system of the UFFO-pathfinder was designed to (i) fit the constraints of the Lomonosov spacecraft, (ii) use all pre-proven technologies and (iii) to be available for fast delivery. The main constraints for inclusion in Lomonosov are $20 \mathrm{~kg}$ total instrument mass and $800 \mathrm{~cm}$ maximum length. As shown in figure 6, the payload consists of two instruments: SMT for rapid coverage of UV/optical sky and the UBAT (UFFO Burst Alert and Trigger Telescope) for $\mathrm{x}$-ray triggers and coarse location. We have designed a small telescope to provide imaging measurements using a gimbal beam-steering system in SMT described above. UBAT is a widefield coded mask camera similar to that of the Swift BAT scaled to fit the available mass and size requirements.

The UFFO-pathfinder has passed space environments test, including thermal, vacuum, shock and vibrations, successfully at National Space Organization (NSPO) of Taiwan in August 2011. The final integration of the flight model to the Lomonosov spacecraft and space environments test is currently under way at a branch of Roscosmos.

\subsection{Slewing Mirror Telescope of UFFO-pathfinder}

As a subsystem of UFFO-pathfinder, the SMT is designed for fast observation of the prompt UV/optical photons from GRBs. The SMT/UFFO-pathfinder uses a gimbal system which provides $1 \mathrm{~s}$ response over the entire FOV of UBAT, $90.2^{\circ} \times 90.2^{\circ}$. Electric motors driving gimbal-mounted mirrors are a fundamentally simple and robust technology. For UFFOpathfinder we used off-the-shelf encoders and motors with sealed bearing systems and have already obtained sub-arcsecond settling over $\pm 90^{\circ}$ with $t<1 \mathrm{~s}$ travel+settle time.

The SMT optics includes a Ritchey-Chretien telescope with a $100 \mathrm{~mm}$ diameter aperture. Its FOV is $17 \times 17 \operatorname{arcmin}^{2}$ and $f$-number is 11.4 . The primary and secondary mirrors were fabricated with the precision of about rms 0.02 waves in wave front error (WFE) and $84.7 \%$ in average reflectivity over $200-650 \mathrm{~nm}$. The entire SMT optics was aligned with the accuracy of rms 0.05 waves in WFE at $632.8 \mathrm{~nm}$ [73]. 


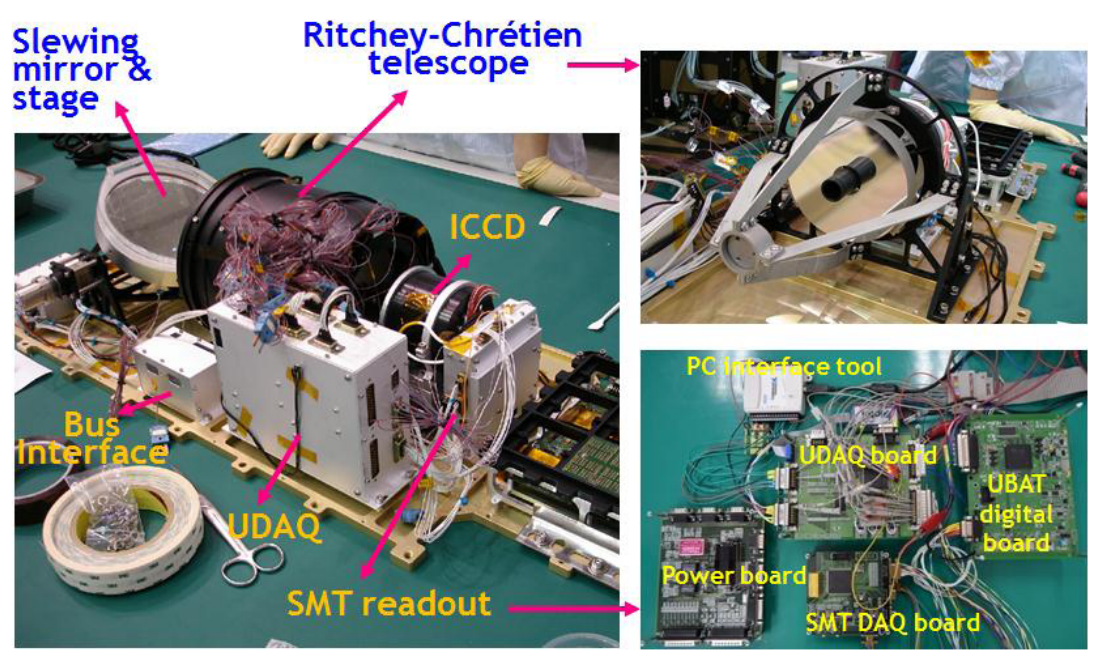

Figure 7. The SMT of the UFFO-pathfinder. The opto-mechanics of the $10 \mathrm{~cm}$ Ritchey-Chretien telescope, the two-axis gimbal mirror and readout electronics are shown, together with the UDAQ box (described in section 6.3). The settle time of the slewing mirror, defined as the time taken for the vibration of a point image to reach the pixel size of ICCD ( 4 arcsec), is 110-340 ms, which implies the difference between two axes.

The focal detector is an intensified charge-coupled device (ICCD) with a pixel size of $4 \times 4 \operatorname{arcsec}^{2}$ and a wavelength sensitive to $200-650 \mathrm{~nm}$. The ICCD operates in photon counting mode and could observe faint objects up to $\sim 19$ magnitude $B$-star in white light per $100 \mathrm{~s}$, assuming the same performance as Swift. The SMT has the readout rate of $20 \mathrm{~ms}$ and can take 50 frames per second.

One of non-trivial issues on UFFO-pathfinder and SMT structures was the reduction of mass, while satisfying structural and functional requirements related to stiffness, strength, dimensional restriction and thermal conduction. The housing was made of carbon fiber. Mirrors and substructure were built through finite element analysis and lightweight engineering. Two identical flight models of SMT shown in figure 7 have been built and delivered.

\subsection{The UFFO Burst Alert and Trigger Telescope of UFFO-pathfinder}

Numerous instruments have used coded-mask aperture shadow cameras (e.g. BeppoSAX [28], HETE-2 [29], Integral [30] and Swift [31]) to determine positions of GRBs. We used the Swift observatory as the benchmark of our x-ray system. Whereas BAT/Swift can localize bursts at $90 \%$ probability to a region 1-4 arcmin, the UBAT will be able to localize bursts at the confidence level of $7 \sigma$ to a region 10 arcmin across, thus contained fully within the FOV of the SMT. Note that, as with most GRB instrument figures of merit, the accuracy applies to some fraction of the brightest bursts, and will vary greatly with faint or rapid bursts. A typical burst will be observed within a $1 \mathrm{~s}$ time bin, tripping a 'rate trigger' in UBAT. The instrument may require longer collection times up to $64 \mathrm{~s}$, depending on the brightness of GRBs. UBAT spends less than a second to determine the position of GRB, using a dedicated field programmable gate array (FPGA) for 'imaging trigger'. Then, SMT starts taking the UV/optical data. 

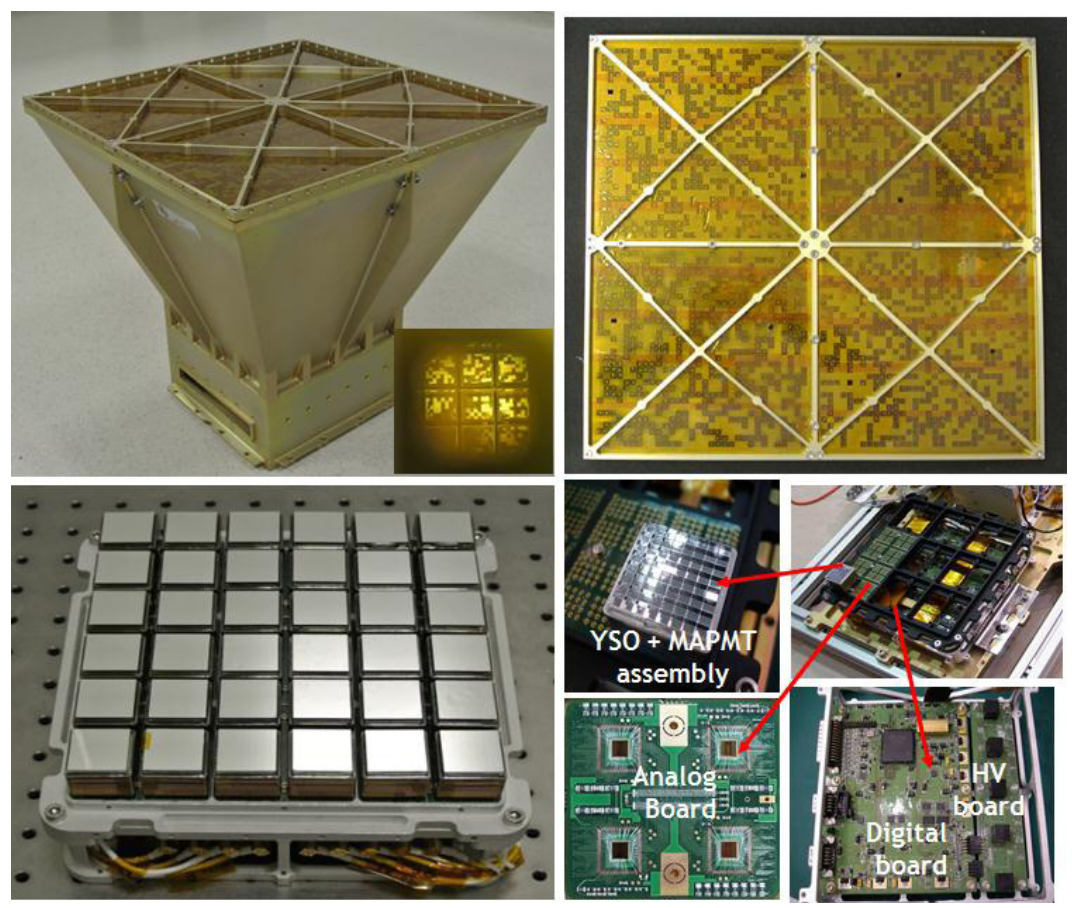

Figure 8. Flight model of the UBAT for the UFFO-pathfinder. It consists of a coded mask (upper right), a hopper and shielding (upper left), a detector (lower left) consisting of YSO crystals, MAPMTs and readout electronics (lower right).

Our science goals and objectives stem from obtaining as large a sample as possible of homogeneously selected GRBs. This translates to an x-ray instrument capable of detecting tens of GRBs per year, and precise localization of GRBs and rapidly triggering the UV/optical observations. Although there are other areas of science in additional information from the high-energy emission of GRBs, we make no specific requirements on energy resolution or flux measurements. We require that the instrument provide only rough flux and duration information for each burst. We anticipate that Fermi [74] with extremely wide sky coverage and high sensitivity and other instruments such as Suzaku [75] will provide additional flux and duration information for a large fraction of bursts.

With the time constraint to meet the launch schedule as well as mass and power constraints for UBAT (only approximately $10 \mathrm{~kg}$ and $10 \mathrm{~W}$ ), we adopted a well-established coded-mask technique similar to Swift BAT but scaled down for the localization of bright, transient x-ray sources. With only $191 \mathrm{~cm}^{2}$ of detecting area, our collaboration has made a viable camera with which we expect to detect dozens of GRBs per year. In order to respond over a wider energy range, e.g. 15-150 keV, however, we used pixellated YSO scintillating crystal read out by 36 64-ch multi-anode photomultiplier tubes (MAPMTs) with 36 64-ch SPACIROC ASICs. Figure 8 shows the integrated UBAT system.

\subsection{The UFFO-pathfinder data acquisition}

The UFFO-pathfinder data acquisition (UDAQ) is in charge of central control of the payload not only with preset commands but upload commands from the ground; interfacing to the spacecraft; 


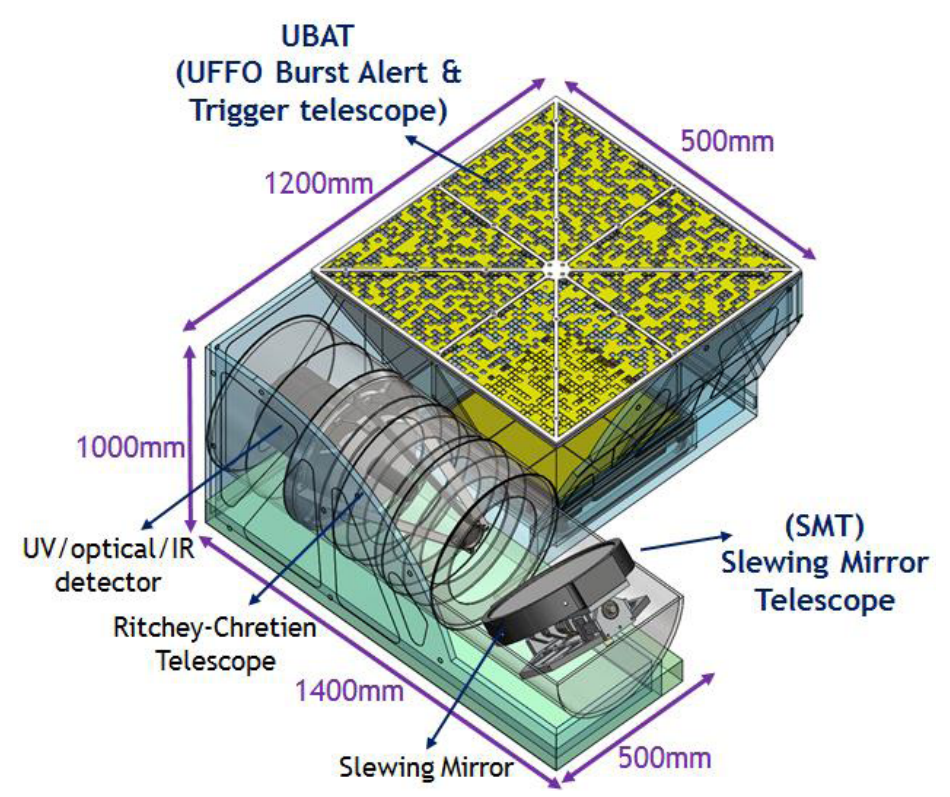

Figure 9. Schematic representation of UFFO-100.

data collection from SMT and UBAT, storing in several NOR flash memories and transfer to the spacecraft. It is also responsible for the monitoring of all housekeeping parameters; calculation of the orbit and recognition of day and night with its photosensors; arbitration and prioritization of triggers from UBAT and BDRG (another GBM/Fermi-like payload of Lomonosov); power management, etc. All of these functions are implemented in an ACTEL FPGA for the low power consumption and fast real-time processing. Trigger calculations with the data from UBAT, including rate trigger and imaging trigger, are also performed in another ACTEL FPGA, which reduces the latency significantly, e.g. below $1 \mathrm{~s}$.

\section{UFFO-100}

Awaiting the completion and launch of the UFFO-pathfinder, the UFFO collaboration has been exploring its next step, a more ambitious project: UFFO-100 (named indicating the mass of payload), based on the same design principle but with total mass larger than $100 \mathrm{~kg}$.

The great instrumental challenge of the UFFO concept is to see changes in the optical light curve on short time scales, which requires short exposures. Therefore, the aperture size of the instrument is the fundamental limitation on both the total number of GRB that may be detected, and the time resolution. GRB gamma-ray light curves, even the longer-duration class, have high amplitude variability at every observed time scale. Comparison of the variability between the gamma-X bands and the optical bands can tell us a great deal about the emission physics at the source. So far, with the most rapid optical measurements available, it is not known whether gamma-X and optical emission correlates, has lags, or perhaps correlates only in certain types of bursts. There are simply not enough short time scale data. The UFFO-100 will answer such an intriguing question of 'What would Swift have seen if it could have responded faster?', with the SMT of an aperture $40 \mathrm{~cm}$ as large as that of Swift, but with several enhancements to make it more sensitive and productive, enabling detections at even shorter time resolution. 
Table 2. Major parameters and expected performance of two UFFO payloads together with Swift.

\begin{tabular}{|c|c|c|c|}
\hline $\begin{array}{l}\text { Parameter or } \\
\text { performance }\end{array}$ & $\begin{array}{l}\text { UFFO-pathfinder/ } \\
\text { Lomonosov }\end{array}$ & UFFO-100 & Swift \\
\hline \multicolumn{4}{|c|}{$X$-ray for localization of GRBs } \\
\hline Detector & $\begin{array}{l}\text { YSO crystal } \\
+ \text { MAPMT }\end{array}$ & $\begin{array}{l}\text { Silicon strip and } \\
{[\text { crystal + (MAPMT or SiPM)] }}\end{array}$ & CdZnTe \\
\hline $\begin{array}{l}\text { Coded mask FOV } \\
\text { (half coded) }\end{array}$ & $\begin{array}{l}90.2 \times 90.2 \\
\operatorname{deg}^{2}(1.8 \mathrm{sr})\end{array}$ & $\begin{array}{l}90.2 \times 90.2 \\
\operatorname{deg}^{2}(1.8 \mathrm{sr})\end{array}$ & $\begin{array}{l}100 \times 60 \\
\operatorname{deg}^{2}(1.4 \mathrm{sr})\end{array}$ \\
\hline Detection area & $191 \mathrm{~cm}^{2}$ & $1024 \mathrm{~cm}^{2}$ & $5240 \mathrm{~cm}^{2}$ \\
\hline Number of pixels & $48 \times 48$ & $64 \times 64$ & $256 \times 128$ \\
\hline Pixel size & $2.8 \times 2.8 \mathrm{~mm}^{2}$ & $2 \times 2 \mathrm{~mm}^{2}$ & $4 \times 4 \mathrm{~mm}^{2}$ \\
\hline Sensitivity & $15-150 \mathrm{keV}$ & $5-300 \mathrm{keV}$ & $15-150 \mathrm{keV}$ \\
\hline Localization error & $10 \operatorname{arcmin}$ & $4 \operatorname{arcmin}$ & $1-4$ arcmin \\
\hline $\begin{array}{l}\text { X-ray collection time/ } \\
\text { GRB position } \\
\text { calculation time }\end{array}$ & $1-64 \mathrm{~s} / 1 \mathrm{~s}$ & $1-64 \mathrm{~s} / 1 \mathrm{~s}$ & $1-64 \mathrm{~s} / 5-7 \mathrm{~s}$ \\
\hline $\begin{array}{l}\text { Number of } \\
\text { GRB localizations } \\
\text { per year }\end{array}$ & $\begin{array}{l}20-30 \text { (duty } \\
\text { cycle and fluence } \\
\text { taken into account) }\end{array}$ & $\sim 70$ & $\sim 100$ \\
\hline \multicolumn{4}{|l|}{ UV/optical/NIR telescope } \\
\hline Type & $\begin{array}{l}\text { Ritchey-Chrétien } \\
\text { + slewing mirror }\end{array}$ & $\begin{array}{l}\text { Modified Ritchey-Chrétien } \\
\text { + slewing mirror }\end{array}$ & Modified Ritchey-Chrétien \\
\hline Aperture & $10 \mathrm{~cm}$ & $40 \mathrm{~cm}$ & $30 \mathrm{~cm}$ \\
\hline FOV & $\begin{array}{l}17 \times 17 \operatorname{arcmin}^{2} \\
\text { over } 70 \times 70 \mathrm{deg}^{2}\end{array}$ & $\begin{array}{l}17 \times 17 \operatorname{arcmin}^{2} \\
\text { over } 90 \times 90 \mathrm{deg}^{2}\end{array}$ & $17 \times 17 \operatorname{arcmin}^{2}$ \\
\hline Wavelength range & $200-650 \mathrm{~nm}$ & $200-1100 \mathrm{~nm}$ & $170-650 \mathrm{~nm}$ \\
\hline Number of pixels & $256 \times 256$ & $256 \times 256$ & $256 \times 256$ \\
\hline Physical pixel scale & $4 \operatorname{arcsec}$ & $4 \operatorname{arcsec}$ & $4 \operatorname{arcsec}$ \\
\hline $\begin{array}{l}\text { Telescope PSF } \\
\text { (centroiding applied) }\end{array}$ & $0.5 \operatorname{arcsec}$ & $0.5 \operatorname{arcsec}$ & $0.5 \operatorname{arcsec}$ \\
\hline Sensitivity & $\begin{array}{l}B=19 \mathrm{mag} \text { in } 100 \mathrm{~s} \\
\text { with } 8 \sigma\end{array}$ & $\begin{array}{l}\text { UV/optical: } B=21.5 \text { and } \\
23.5 \text { mag in } 100 \mathrm{~s} \text { with } \\
5 \sigma \text { for UV/optical } \\
\text { and NIR, respectively }\end{array}$ & $\begin{array}{l}B=24 \mathrm{mag} \text { in } 1000 \mathrm{~s} \\
\text { with } 5 \sigma\end{array}$ \\
\hline $\begin{array}{l}\text { Observation } \\
\text { start time after } \\
\text { x-ray trigger }\end{array}$ & $1 \mathrm{~s}$ & $0.01-1 \mathrm{~s}$ & $\begin{array}{l}40-200 \text { s, typically } \\
80 \text { s (see figure } 1 \text { ) }\end{array}$ \\
\hline $\begin{array}{l}\text { Number of } \\
\text { UV/optical observations }\end{array}$ & $\begin{array}{l}\text { 10-20 (estimated from } \\
\text { the extrapolation of } \\
\text { early light curves) }\end{array}$ & $30-40$ & $\sim 40$ \\
\hline
\end{tabular}

Although some enhancements may be restricted by details of payload requirements, UFFO100 would afford a $1024 \mathrm{~cm}^{2}$ x-ray camera with improved detector technology. The MMA technology could be integrated with the SMT, as shown in figure 4. The UV/optical and NIR cameras, both with 17 arcmin fields, will use the incoming beam from the 40 aperture SMT 
after being split from a dichroic. Much of the instrumentation, particularly the electronics, will be built on the heritage of UFFO-pathfinder. The pathfinder basic telescope design, fast-mode beam steering, spacecraft bus interface and data acquisition system architecture will be shared with UFFO-100. The key components and the dimensions of UFFO-100 are shown in figure 9. We expect UFFO-100 to be flown as one of the scientific payloads of the Russian Resurs-P3 satellite in 2018. The comparison of UFFO-100 to UFFO-pathfinder, together with Swift, is shown in table 2 .

\section{Summary}

We propose two space missions implementing the UFFO approach in order to investigate a new area of gamma-ray burst phase space both quantitatively and qualitatively. The UFFO equipped with SMT has an extraordinary capability by permitting the first ever systematic study of GRB UV/optical/NIR emission, for example $1 \mathrm{~s}$ after trigger for UFFO-pathfinder and far earlier than $1 \mathrm{~s}$ after trigger for UFFO-100. Our fundamental science objective is to use our ability to probe this new, very early emission parameter space to make measurements of and thoroughly investigate the rise phase of GRB, which is so far only occasionally observed. In the time domain, this improves on Swift's response by several orders of magnitude. In the spectral domain, we will improve on Swift's sensitivity by $\sim 2.5$ mag (assuming the power-law light curve extends at very early times), and we expect to detect afterglow components that are invisible to Swift because of extinction.

The UFFO-pathfinder has now entered the final stage of completion, heading for launch onboard Lomonosov satellite in 2013. The UFFO-100 will be improved with modest size and launched in 2018. Both payloads are small and limited, yet remarkably powerful microobservatories for rapid optical response within $1 \mathrm{~s}$ after x-ray trigger to bright GRBs. Their sub-minute measurements of the optical emission of dozens of GRBs each year will result in a more rigorous test of current internal shock models, probe the extremes of bulk Lorentz factors, provide the first early and detailed measurements of fast-rise GRB optical light curves and possibly test the prospect of GRBs as extreme $z$ cosmological probes. We foresee not only such exciting outcomes but the proof-of-principle of this new approach for future GRB telescopes. After Swift, UFFO-100 will continue to provide numerous GRBs for follow-up studies by the optical ground based multi-wavelength communities. If other Swift-like GRB missions were to fly in the near future, their sensitivity would complement UFFO's rapid response.

\section{Acknowledgments}

This work was supported by the Creative Research Initiatives program (RCMST) of MEST/NRF. IP, EL, GS and HL are members of IEU, which is funded by the WCU program (R32-2009-000-10130-0). VL and ACT acknowledge financial support from the Spanish MINECO through project number AYA 2009-14000-C03-01/ESP and PC Taiwan's National Science Council Vanguard Program (100-2119-M-002-025) and the MP program of development of Lomonosov Moscow State University. We acknowledge the NSPO and the Nauchno-issledovatelskij Institut Elektromehaniki (NIIEM) for their support with numerous tests of our instruments for space qualification. Our special thanks are due to H S Yang of KRISS and H K Lee of Samsung Electronics for their assistance in the design and manufacture of SMT mirrors. Also, we thank Z L Uhm for the figure on the bulk Lorentz factor measurements. 


\section{References}

[1] Salvaterra R et al 2009 Nature 4611258

[2] Lamb D Q and Reichart D E 2000 Astrophys. J. 5361

[3] Bromm V and Loeb A 2006 Astrophys. J. 642382

[4] Kistler M D et al 2009 Astrophys. J. 705 L104

[5] Amati L et al 2008 Mon. Not. R. Astron. Soc. 391577

[6] Ghirlanda G et al 2006 New J. Phys. 8123

[7] Panaitescu A and Vestrand W 2008 Mon. Not. R. Astron. Soc. 387497

[8] Kocevski D 2012 Astrophys. J. 747146

Shahmoradi A and Nemiroff R J 2011 Mon. Not. R. Astron. Soc. 4111843

Bernardini M G, Margutti R, Zaninoni E and Chincarini G 2012 Mon. Not. R. Astron. Soc. 4251199

[9] Perlmutter S et al 1999 Astrophys. J. 51565

[10] Percival W J et al 2010 Mon. Not. R. Astron. Soc. 4012148

[11] Munshi D et al 2008 Phys. Rep. 46267

[12] Zhang B et al 2009 Astrophys. J. 7031696

[13] Greiner J et al 2011 Astron. Astrophys. 52610

[14] Cline D B, Otwinowski S, Czerny B and Janiuk A 2011 arXiv:1105.5363

[15] Bloom J S et al 2011 Science 333203

Burrows D N et al 2011 Nature $\mathbf{4 7 6} 421$

[16] Campana S et al 2006 Nature 4421008

[17] Waxman E 2006 Nucl. Phys. B (Supp. Proc.) 15146

[18] Cutler C and Thorne K S 2002 arXiv:gr-qc/0204090

[19] Abu-Zayyad T et al 2012 Nucl. Instrum. Methods A 68987 Tokuno H et al 2012 Nucl. Instrum. Methods A 67654

[20] Abraham J et al 2004 Nucl. Instrum. Methods A 52350

[21] Panasyuk M I et al 2012 J. Cosmol. 187964

[22] Takahashi Y 2009 New J. Phys. 11065009

[23] Halzen F and Klein S R 2010 Rev. Sci. Instrum. 81081101

[24] Gorham P W et al 2009 Astropart. Phys. 3210

[25] Abramovici A et al 1992 Science 256325

[26] Pau A-S et al 2012 arXiv:1201.3621

[27] Fishman G J 1994 Astrophys. J. Suppl. 92229

[28] Boella G et al 1997 Astron. Astrophys. Suppl. 122299

[29] Ricker G et al 2002 Astrophys. J. 571 L127

[30] Winkler C et al 2003 Astron. Astrophys. 411 L1

[31] Gehrels N et al 2004 Astrophys. J. 6111005

[32] Perna R et al 2003 Astrophys. J. 585775

Oates S R et al 2009 Mon. Not. R. Astron. Soc. 395490

Perley D A et al 2010 Mon. Not. R. Astron. Soc. 4062473

[33] Paul J et al 2011 C. R. Phys. 12298

[34] Roming P W A et al 2012 Mem. Soc. Astron. Suppl. 21155

[35] Produit N et al 2005 Nucl. Instrum. Methods A 550616

[36] Costa E et al 1997 Nature 387783

Kann D A et al 2011 Astrophys. J. 73496

[37] Ellis J et al 2006 Astropart. Phys. 25402

[38] Kostelecky V A and Mewes M 2008 Astrophys. J. 689 L1

[39] Park I H et al 2009 arXiv:0912.0773

[40] Barthelmy S D 2004 Proc. SPIE 5165175 
[41] Akerlof C W et al 2003 Publ. Astron. Soc. Pac. 115132

[42] Vestrand W T et al 2002 Proc. SPIE 4845126

[43] Bloom J S et al 2006 Astron. Soc. Pac. Conf. Ser. 351751

[44] Williams G G et al 2004 AIP Conf. Proc. 727723

[45] Jelinek M et al 2010 Adv. Astron. 432172 http://bootes.iaa.es

[46] Akerlof C et al 1999 Nature 398400

[47] Racusin J L et al 2008 Nature 455183

[48] Beskin G et al 2010 Adv. Astron. 171569

[49] Burd A et al 2005 New Astron. 10409

[50] Aptekar R L et al 1995 Space Sci. Rev. 71265

[51] Roming P et al 2005 Space Sci. Rev. 12095

[52] Kim M et al 2009 J. Micromech. Microeng. 19035014

[53] Park J H et al 2008 Opt. Express 1620249

[54] Nam S et al 2008 Nucl. Instrum. Methods A 588197

Yoo B W et al 2009 Opt. Express 173370

[55] van Paradijs J et al 1997 Nature 386686

[56] Tanvir N R et al 2009 Nature 4611254

[57] Kouveliotou C et al 1993 Astrophys. J. 413 L101

[58] Zhang F-W et al 2012 Astrophys. J. 75088

[59] MacFadyen A I and Woosley S E 1999 Astrophys. J. 524262

[60] Nakar E 2007 Phys. Rep. 442166

[61] Norris J P and Bonnell J T 2006 Astrophys. J. 643266

[62] Perley D A et al 2009 Astrophys. J. 1381690

[63] Groot P J et al 1998 Astrophys. J. 502 L123

[64] Vestrand W T et al 2005 Nature 435178

[65] Molinari E et al 2007 Astron. Astrophys. 46913

[66] Piran T 2004 Rev. Mod. Phys. 761143

[67] Stoldosky L 2000 Phys. Lett. B 47361

[68] Robertson B E and Ellis R S 2011 arXiv:1109.0990

[69] Greiner J et al 2011 Astron. Astrophys. 52610

[70] Perna R et al 2003 Astrophys. J. 585775

[71] Oates S R et al 2009 Mon. Not. R. Astron. Soc. 395490

Perley D A et al 2010 Mon. Not. R. Astron. Soc. 4062473

[72] Grossan B et al 2012 SPIE Astron. Telesc. Instrum. 8443 84432R

[73] Jeong S et al 2013 Opt. Express 212263

[74] Atwood W B et al 2009 Astrophys. J. 6971071

Meegan C et al 2009 Astrophys. J. $\mathbf{7 0 2} 791$

[75] Mitsuda K et al 2007 Publ. Astron. Soc. Japan 591 